\title{
THE EFIMOV EFFECT OF THREE-BODY SCHRÖDINGER OPERATORS: ASYMPTOTICS FOR THE NUMBER OF NEGATIVE EIGENVALUES
}

\author{
HIDEO TAMURA
}

\section{Introduction}

The Efimov effect is one of the most remarkable results in the spectral theory for three-body Schrödinger operators. Roughly speaking, the effect will be explained as follows: If all three two-body subsystems have no negative eigenvalues and if at least two of these two-body subsystems have resonance states at zero energy, then the three-body system under consideration has an infinite number of negative eigenvalues accumulating at zero. This remarkable spectral property was first discovered by Efimov [1] and the problem has been discussed in several physical journals. For related references, see, for example, the book [3]. The mathematically rigorous proof of the result has been given by the works $[4,8,9]$. The aim of the present work is to study the asymptotic distribution of these negative eigenvalues below zero (bottom of essential spectrum). Denote by $N(E), E>0$, the number of negative eigenvalues less than $-E$. Then the main result obtained here is, somewhat loosely stating, that $N(E)$ behaves like $|\log E|$ as $E \rightarrow 0$. We first formulate precisely the main theorem and then make a brief comment on the recent related result obtained by Sobolev [7].

We consider a system of three particles with masses $m_{j}>0,1 \leq j \leq 3$, which move in the three-dimensional space $R^{3}$ and interact with each other through a pair potential $V_{j k}\left(r_{j}-r_{k}\right), 1 \leq j<k \leq 3$, where $r_{j} \in R^{3}$ denotes the position vector of the $j$-th particle. For such a system, the energy Hamiltonian $H$ (three-body Schrödinger operator) takes the form

$$
H=H_{0}+V, \quad V=\sum_{1 \leq j<k \leq 3} V_{j k}\left(r_{j}-r_{k}\right),
$$

in the center-of-mass frame, where $H_{0}$ denotes the free Hamiltonian. Both the

Received May 28, 1992. 
Hamiltonians $H_{0}$ and $H$ are regarded as an operator acting on the space $L^{2}\left(R^{6}\right)$ and are represented in various forms according to the choice of the Jacobi coordinates. All the pair potentials $V_{j k}$ are assumed to satisfy the following condition:

$(V)_{\rho} V_{\jmath k}(x), x \in R^{3}$, is real-valued and has the decay property

$$
\left|V_{j k}(x)\right| \leq C(1+|x|)^{-\rho} \text { for some } \rho>2 .
$$

Throughout the whole exposition, the constant $\rho$ is used with the meaning ascribed above. This assumption implies that the Hamiltonian $H$ formally defined above admits a unique self-adjoint realization $L^{2}\left(R^{6}\right)$. We denote by the same notation $H$ this self-adjoint realization.

We use letters $\alpha, \beta, \gamma$ and $\delta$ to denote one of three pairs $(j, k)$ with $1 \leq j$ $<k \leq 3$. For given pair $\alpha=(j, k)$, we define the reduced mass $m_{a}$ through the relation $1 / m_{a}=1 / m_{j}+1 / m_{k}$ and the two-body subsystem Hamiltonian $H^{\alpha}$ as

$$
H^{\alpha}=-\Delta / 2 m_{a}+V_{a}, \quad V_{a}(x)=V_{j k}(x) \text {, on } L^{2}\left(R_{x}^{3}\right) \text {. }
$$

We further assume that all the two-body subsystem Hamiltonians $H^{\alpha}$ have the following spectral properties:

(H.1) $H^{\alpha}$ has no negative bound state energies.

(H.2) $H^{\alpha}$ has a resonance state at zero energy.

Roughly speaking, assumption (H.2) means that the equation $H^{\alpha} \varphi=0$ has a solution behaving like $\varphi(x) \sim|x|^{-1}, x \in R^{3}$, at infinity. It should be noted that $\varphi$ is not an eigenstate at zero energy of $H^{\alpha}$. By the $H V Z$ theorem ([5]), it follows from (H.1) that the three-body Hamiltonian $H$ has its essential spectrum in the interval $[0, \infty)$ and its discrete spectrum in $(-\infty, 0)$. If, in addition, (H.2) is satisfied, then $H$ has an infinite number of negative eigenvalues accumulating at zero. As stated above, this spectral property is known as the Efimov effect. The aim here is to study the asymptotic distribution of such negative eigenvalues accumulating at zero. The main theorem is formulated as follows.

Theorem 1. Assume that $(V)_{\rho}$, (H.1) and (H.2) are fulfilled. Let $N(E), E>0$, be the number of negative eigenvalues less than $-E$ of $H$ with repetition according to their multiplicities. Then $N(E)$ obeys the following asymptotic formula:

$$
N(E)=C_{0}|\log E|(1+o(1)), \quad E \rightarrow 0,
$$

for some $C_{0}>0$. 
Remark 1. We should make some comments on the leading coefficient $C_{0}$ in the asymptotic formula. This constant $C_{0}$ does not depend on the pair potentials $V_{j k}$ and is given as a positive function of only the ratios $m_{j} / m_{k}$ between the masses. As is seen in the proof of the theorem, it is determined from an eigenvalue asymptotics for a certain compact integral operator and is in general difficult to write down in the explicit form. In the special case with identical masses, $C_{0}$ is determined as $C_{0}=\lambda / 2 \pi$ with the unique positive root $\lambda>0$ of the equation

$$
\lambda=2^{3} \cdot 3^{-1 / 2}(\sinh \pi \lambda / 6) /(\cosh \pi \lambda / 2) .
$$

Remark 2. (1) The following result can be also obtained in the course of proof: If at most one of subsystem Hamiltonians has a resonance state at zero energy, then $H$ has only a finite number of negative eigenvalues; $N(E)=O(1), E$ $\rightarrow 0$. This result asserts the finiteness of discrete spectrum below the bottom of essential spectrum, even if the bottom coincides with a three-cluster threshold energy. (2) As previously stated, $H$ has in general an infinite number of negative eigenvalues accumulating at zero except for a certain special case, if only two subsystem Hamiltonians have a resonance state at zero energy. The theorem above can be extended to such a case. We will discuss briefly this problem in the last section.

The asymptotic formula in the theorem has been first established by Sobolev [7] under the main assumption that pair potentials are non-positive and have the decay property $(V)_{\rho}$ with $\rho>3$, and also the above properties of the leading coefficient $C_{0}$ has been investigated in detail there. In the present work, we improve slightly this result with emphasis on the following cases: (1) pair potentials are not necessarily assumed to be non-positive; (2) pair potentials have the weak decay property $(V)_{\rho}$ with $\rho>2$. The proof of the theorem is, in principle, based on the same idea developed by [7] but the arguments undergo a slight change in many aspects, if the non-positivity assumption of pair potentials is not necessarily assumed. We first reduce the problem under consideration to the study on the eigenvalue asymptotics for a certain compact integral operator. The reduction is made by use of the results on the behavior at low energies of two-body resolvents. After reduction, we apply the results obtained in [7] to calculate the leading coefficient of asymptotic formula for eigenvalues of such an integral operator.

The method here applies also to the problem on the eigenvalue asymptotics in the coupling limit. Let $H$ be defined by (0.1). Suppose that $H$ fulfills all the 
assumptions in Theorem 1. We consider the three-body Hamiltonian

$$
H(\lambda)=H-\lambda V=H_{0}+(1-\lambda) V \text { on } L^{2}\left(R^{6}\right)
$$

with a coupling constant $\lambda, 0<\lambda \ll 1$, small enough. The Hamiltonian $H(\lambda)$ has only a finite number of negative eigenvalues for $\lambda>0$. The theorem below gives the asymptotic formula as $\lambda \rightarrow 0$ for the number of such negative eigenvalues.

THEOREM 2. Suppose that the three-body Hamiltonian $H$ fulfills assumptions $(V)_{\rho}$, (H.1) and (H.2). Let $H(\lambda), 0<\lambda \ll 1$, be as above. Denote by $N_{0}(\lambda)$ the number of negative eigenvalues of $H(\lambda)$. Then one has

$$
N_{0}(\lambda)=2 C_{0}|\log \lambda|(1+o(1)), \quad \lambda \rightarrow 0,
$$

with the same positive constant $C_{0}$ as in Theorem 1 .

\section{§1. Low energy analysis for two-body resolvents}

In this section we formulate the result on the behavior at low energies of two-body resolvents, which is used as a basic tool to prove Theorems 1 and 2 . Throughout the section, we work in the space $L^{2}=L^{2}\left(R_{x}^{3}\right)$ and denote by $\langle$, the $L^{2}$ scalar product in this space.

We begin by defining precisely the resonance state at zero energy. Let $T=-\Delta+V_{0}$ be the two-body Schrödinger operator acting on $L^{2}$. We assume that the potential $V_{0}(x)$ has the decay property $(V)_{\rho}$ and that the operator $T$ has the spectral properties (H.1) and (H.2). We now consider the equation $T \varphi=0$. This equation can be put into the integral equation

$$
\varphi(x)=-(1 / 4 \pi) \int|x-y|^{-1} V_{0}(y) \varphi(y) d y,
$$

where the integration with no domain attached is taken over the whole space. This abbreviation is used throughout. Equation (1.1) is considered in the weighted $L^{2}$ space $L_{-s}^{2}=L^{2}\left(R_{x}^{3} ;\langle x\rangle^{-2 s} d x\right),\langle x\rangle=\left(1+|x|^{2}\right)^{1 / 2}$, with $s>1 / 2, s$ being taken close enough to $1 / 2$. If $\varphi \in L_{-s}^{2}$ solves the equation (1.1), then it is easily seen that $\varphi$ behaves like

$$
\begin{aligned}
& \varphi(x)=-(1 / 4 \pi)\left\langle V_{0}, \varphi\right\rangle|x|^{-1}+O\left(|x|^{-\rho+1}\right), \\
& (\partial / \partial|x|) \varphi(x)=(1 / 4 \pi)\left\langle V_{0}, \varphi\right\rangle|x|^{-2}+O\left(|x|^{-\rho}\right)
\end{aligned}
$$

as $|x| \rightarrow \infty$. We classify the solution to (1.1) into two kinds of solutions accord- 
ing as the scalar product $\left\langle V_{0}, \varphi\right\rangle$ vanishes or not. We say that $\varphi$ is the resonance state at zero energy of $T$, when $\left\langle V_{0}, \varphi\right\rangle \neq 0$ is satisfied. Thus the resonance state $\varphi$ behaves like $\varphi(x) \sim|x|^{-1}$ as $|x| \rightarrow \infty$ and hence $\varphi \notin L^{2}$ is not a bound state at zero energy. On the other hand, if $\left\langle V_{0}, \varphi\right\rangle=0$ is satisfied, then we have $\varphi(x)=O\left(|x|^{-\rho+1}\right)$ and hence it follows from (1.1) that $\varphi(x)=O\left(|x|^{-\rho+1-\varepsilon}\right)$, $\varepsilon=\rho-2>0$. We repeat this argument to obtain that $\varphi(x)=O\left(|x|^{-2}\right)$, so that $\varphi$ is in $L^{2}$ and becomes a bound state at zero energy of $T$. Conversely, if $\varphi$ is a bound state at zero energy, then we can easily see that $\varphi$ satisfies the relation $\left\langle V_{0}, \varphi\right\rangle=0$ and hence the resonance state at zero energy is non-degenerate. The following lemma is obtained as an immediate consequence of Theorem XIII.46, [5] and Theorem A.3.1, [6].

Lemma 1.1. Suppose that $T$ fulfills $(V)_{\rho}$, (H.1) and (H.2). Then $T$ cannot have a bound state at zero energy, bottom of its spectrum, and hence has only a resonance state.

Assumption $(V)_{\rho}$ enables us to choose a non-negative potential $U_{0} \geq 0$ satisfying $(V)_{\rho}$ so that

$$
W_{0}(x)=U_{0}(x)-V_{0}(x) \geq U_{0}(x) / 2 \geq 0 .
$$

If $V_{0}$ is non-positive, then we can take $U_{0}=0$. Let $S$ be the Schrödinger operator with potential $U_{0}$

$$
S=-\Delta+U_{0} \text { on } L^{2}\left(R_{x}^{3}\right)
$$

and denote the resolvent of $S$ as $R\left(d^{2} ; S\right)=\left(S+d^{2}\right)^{-1}$ for $d>0$. Since $U_{0}$ is non-negative, $R(0 ; S)$ can be also defined as a bounded operator from $L_{s}^{2}$ into $L_{-s}^{2}$ for any $s>1$ and the generalized eigenfunction $\theta_{0}(x)$ at zero energy of $S$ is obtained as a unique solution to the Lippmann-Schwinger equation. This eigenfunction $\theta_{0}$ is easily seen to obey the following bounds as $|x| \rightarrow \infty$ :

$$
\theta_{0}(x)=1+o(1), \quad(\partial / \partial|x|) \theta_{0}(x)=o\left(|x|^{-1}\right) .
$$

We now define the operator $A(d): L^{2} \rightarrow L^{2}$ by

$$
A(d)=\mathrm{Id}-W_{0}^{1 / 2} R\left(d^{2} ; S\right) W_{0}^{1 / 2}, \quad d \geq 0,
$$

Id being the identity operator. It should be noted that this operator can be defined even for $d=0$. Denote by $\sum_{1}$ the kernel of $A(0)$. Since $W_{0}^{1 / 2} R(0 ; S) W_{0}^{1 / 2}$ is a compact operator, the kernel $\Sigma_{1}$ is of finite dimension.

We can show that $\Sigma_{1}$ is a one-dimensional space, if $T$ has a resonance state at zero energy. To see this, we investigate the relation between the kernels of $T$ 
and $A(0)$. Let $\varphi$ be a solution to (1.1). Then $\varphi$ obeys the equation

$$
T \varphi=S \varphi-W_{0} \varphi=0
$$

and hence $\phi \in L^{2}$ defined as $\phi=W_{0}^{1 / 2} \varphi$ belongs to $\Sigma_{1}$. We take the scalar product of $\theta_{0}$ with (1.7). If we take account of the asymptotic behaviors (1.2) and (1.5), then we obtain, by the Green formula, the relation

$$
\left\langle V_{0}, \varphi\right\rangle=-\left\langle\theta_{0} W_{0}^{1 / 2}, \phi\right\rangle
$$

for $\varphi$ and $\phi$ as above. Conversely, if $\phi$ belongs to $\Sigma_{1}$, then we see, repeating a similar argument, that $\varphi$ defined as

$$
\varphi=R(0 ; S) W_{0}^{1 / 2} \phi=W_{0}^{-1 / 2} \phi
$$

solves the equation (1.1) and satisfies the same relation as in (1.8). Thus, if $T$ has a resonance state at zero energy, it follows from Lemma 1.1 that $\Sigma_{1}$ is a one-dimensional space. Denote by $\phi_{1} \in L^{2}$ the normalized function spanning $\Sigma_{1}$. Then $\phi_{1}$ satisfies

$$
\left\langle\theta_{0}, W_{0}^{1 / 2} \phi_{1}\right\rangle \neq 0
$$

by (1.8) and falls off with order $O\left(|x|^{-1-\rho / 2}\right)$ by (1.9).

Let $\Sigma_{2}$ be the orthogonal complement of $\Sigma_{1}$. We decompose the space $L^{2}=$ $L^{2}\left(R_{x}^{3}\right)$ into the orthogonal sum $L^{2}=\Sigma_{1} \oplus \Sigma_{2}$ and denote by $P_{j}, 1 \leq j \leq 2$, the orthogonal projections onto $\sum_{j}$. Since $\phi_{1}$ behaves like $\phi_{1}(x)=O\left(|x|^{-1-\rho / 2}\right)$ at infinity, we can obtain the following lemma.

Lemma 1.2. The orthogonal projection $P_{1}$ can be extended to a bounded operator from $L_{-s}^{2}$ into $L_{s}^{2}$ for any $s, 1 / 2<s<(\rho-1) / 2$.

We study the behavior as $d \rightarrow 0$ of $A(d)$ defined by (1.6). To do this, we here introduce new notations. A bounded operator $T(d), 0<d \ll 1$, acting on $L^{2}$ is said to be of class $\operatorname{Op}\left(d^{\nu}\right)$, if its operator norm obeys the bound $\|T(d)\|=$ $O\left(d^{\nu}\right)$ as $d \rightarrow 0$. When the difference $T_{1}(d)-T_{2}(d)$ is of class $O \mathrm{p}\left(d^{\nu}\right)$ for given two operators $T_{1}(d)$ and $T_{2}(d)$, we denote this relation as $T_{1}(d)=T_{2}(d)+$ $\mathrm{Op}\left(d^{\nu}\right)$.

Lemma 1.3. Suppose that $T$ fulfills $(V)_{\rho}$, (H.1) and (H.2). The operator $A(d)$ defined by (1.6) has the following properties.

(1) Let $\varepsilon, 0<\varepsilon \ll 1$, be fixed arbitrarily. Then there exist positive constants $c_{\varepsilon}$ and $c_{\varepsilon}^{\prime}$ such that 


$$
c_{\varepsilon} \mathrm{Id} \leq A(d) \leq c_{\varepsilon}^{\prime} \mathrm{Id}, \quad d \geq \varepsilon
$$

in the form sense.

(2) Let $P_{j}, 1 \leq j \leq 2$, be as above and let $\phi_{1} \in L^{2}$ be the normalized function spanning the range of $P_{1}$, the range being of one dimension. Define $A_{j k}(d), 1 \leq$ $j, k \leq 2$, as $A_{j k}(d)=P_{j} A(d) P_{k}$. Then:

(i ) $A_{22}(d) \in \mathrm{Op}\left(d^{0}\right)$ and $A_{22}(d) \geq c_{2} P_{2}$ for some $c_{2}>0$.

(ii) $A_{12}(d) \in \mathrm{Op}\left(d^{\nu}\right)$ for some $\nu>1 / 2$.

(iii) There exists $\nu>1$ such that $A_{11}(d)=\sigma_{1} d P_{1}+\mathrm{Op}\left(d^{\nu}\right)$ with

$$
\left.\sigma_{1}=\left|\left\langle\theta_{0}, W_{0}^{1 / 2} \phi_{1}\right\rangle\right|^{2} / 4 \pi\right\rangle 0
$$

This lemma plays an important role in proving the main theorems. We proceed with the argument, accepting the lemma as proved. The proof is given in section 4 after completing the proof of Theorem 1.

Remark 1.4. An similar argument applies to the Schrödinger operator $T=-\Delta / 2 m+V_{0}$ with reduced mass $m$. For such an operator, the constant $\sigma_{1}$ in the lemma is given as

$$
\sigma_{1}=2^{-1 / 2} \pi^{-1} m^{3 / 2}\left|\left\langle\theta_{0}, W_{0}^{1 / 2} \phi_{1}\right\rangle\right|^{2},
$$

where $\theta_{0}$ is the generalized eigenfunction at zero energy of $S=-\Delta / 2 m+U_{0}$, $U_{0}$ being chosen to satisfy (1.3), and $\phi_{1} \in L^{2}$ is the normalized function constructed for the operator $S$. This can be easily verified by a simple scale transformation.

\section{§2. Three-body system}

From now on, we always assume that $(V)_{\rho}$, (H.1) and (H.2) are fulfilled. In this section we introduce several basic notations used throughout the proof of the main theorems.

Let $\alpha=(j, k)$ be given pair and let $l, l \neq j, k$, be the index by which the third particle is labelled. Then the Jacobi coordinates associated with $\alpha$ are defined as

$$
x_{\alpha}=r_{j}-r_{k}, \quad y_{\alpha}=r_{l}-\left(m_{j} r_{j}+m_{k} r_{k}\right) /\left(m_{j}+m_{k}\right) .
$$

We denote by $\left(p_{\alpha}, q_{\alpha}\right) \in R^{3 \times 2}$ the coordinates dual to $\left(x_{\alpha}, y_{\alpha}\right)$. In this coordinate system, the symbol $H_{0}\left(p_{\alpha}, q_{\alpha}\right)$ of the three-body free Hamiltonian $H_{0}$ is described as 


$$
H_{0}\left(p_{\alpha}, q_{\alpha}\right)=\left|p_{\alpha}\right|^{2} / 2 m_{\alpha}+\left|q_{\alpha}\right|^{2} / 2 n_{\alpha},
$$

where $m_{\alpha}$ again denotes the reduced mass associated with $\alpha$ and $n_{\alpha}$ is defined through the relation $1 / n_{\alpha}=1 / m_{l}+1 /\left(m_{j}+m_{k}\right)$. Let $\beta \neq \alpha$ be another pair. Then a simple calculation yields

$$
p_{\alpha}=\kappa^{\alpha \alpha} q_{\alpha}+\kappa^{\alpha \beta} q_{\beta}, \quad p_{\beta}=\kappa^{\beta \alpha} q_{\alpha}+\kappa^{\beta \beta} q_{\beta},
$$

where the coefficients $\kappa^{\alpha \alpha}, \kappa^{\beta \alpha}, \kappa^{\alpha \beta}$ and $\kappa^{\beta \beta}$ are explicitly expressed in terms of the masses $m_{j}, 1 \leq j \leq 3$, and, in particular, $\kappa^{\beta \alpha}$ and $\kappa^{\alpha \beta}$ satisfy $\left|\kappa^{\beta \alpha}\right|=\left|\kappa^{\alpha \beta}\right|=1$. We sometimes denote by $H_{0}\left(q_{\alpha}, q_{\beta}\right)$ the symbol representation for $H_{0}$ in the coordinate system $\left(q_{\alpha}, q_{\beta}\right)$. We further define the cluster Hamiltonian $H_{\alpha}$ as

$$
H_{\alpha}=H_{0}+V_{\alpha}, \quad V_{\alpha}=V_{j k} \quad \text { on } L^{2}\left(R^{6}\right) .
$$

The base space $L^{2}\left(R^{6}\right)$ is decomposed as the tensor product

$$
L^{2}\left(R^{6}\right)=L^{2}\left(R^{3} ; d x_{\alpha}\right) \otimes L^{2}\left(R^{3} ; d y_{\alpha}\right)
$$

and hence the Hamiltonian $H_{\alpha}$ is represented as

$$
H_{\alpha}=H^{\alpha} \otimes \mathrm{Id}+\mathrm{Id} \otimes T_{\alpha} \text { on } L^{2}\left(R^{3} ; d x_{\alpha}\right) \otimes L^{2}\left(R^{3} ; d y_{\alpha}\right),
$$

where $H^{\alpha}$ again denotes the two-body subsystem Hamiltonian associated with $\alpha$ and $T_{\alpha}$ is given as

$$
T_{\alpha}=-\Delta / 2 n_{\alpha} \quad \text { on } \quad L^{2}\left(R^{3} ; d y_{\alpha}\right)
$$

We now choose a non-negative potential $U_{\alpha}=U_{\alpha}\left(x_{\alpha}\right) \geq 0$ to satisfy a relation similar to (1.3)

$$
W_{\alpha}\left(x_{\alpha}\right)=U_{\alpha}\left(x_{\alpha}\right)-V_{\alpha}\left(x_{\alpha}\right) \geq U_{\alpha}\left(x_{\alpha}\right) / 2 \geq 0
$$

and define the Hamiltonians $K^{\alpha}$ and $K_{\alpha}$ as

$$
\begin{aligned}
& K^{\alpha}=-\Delta / 2 m_{\alpha}+U_{\alpha} \text { on } L^{2}\left(R^{3} ; d x_{\alpha}\right), \\
& K_{\alpha}=K^{\alpha} \otimes \operatorname{Id}+\operatorname{Id} \otimes T_{\alpha} \text { on } L^{2}\left(R^{3} ; d x_{\alpha}\right) \otimes L^{2}\left(R^{3} ; d y_{\alpha}\right) .
\end{aligned}
$$

In a similar way to (1.6), we also define $A\left(d ; K^{\alpha}\right): L^{2}\left(R^{3} ; d x_{\alpha}\right) \rightarrow L^{2}\left(R^{3} ; d y_{\alpha}\right)$ as

$$
A\left(d ; K^{\alpha}\right)=\mathrm{Id}-W_{\alpha}^{1 / 2}\left(K^{\alpha}+d^{2}\right)^{-1} W_{a}^{1 / 2}, \quad d \geq 0,
$$

and denote by $P_{j}^{\alpha}, 1 \leq j \leq 2$, the orthogonal projections associated with $A\left(0 ; K^{\alpha}\right)$, which are constructed in the same way as $P_{j}$ in section 1 . We further denote by $\theta_{0}^{\alpha}=\theta_{0}^{\alpha}\left(x_{a}\right)$ the generalized eigenfunction at zero energy of $K^{\alpha}$ and by 
$\phi_{1}^{\alpha} \in L^{2}\left(R^{3} ; d x_{\alpha}\right)$ the normalized function spanning the range of $P_{1}^{\alpha}$. The operator $A\left(d ; K^{\alpha}\right)$ defined above preserves the same properties as in Lemma 1.3 (see also Remark 1.4) and, in particular, we have

$$
P_{1}^{\alpha} A\left(d ; K^{\alpha}\right) P_{1}^{\alpha}=\sigma_{\alpha} d P_{1}^{\alpha}+\mathrm{Op}\left(d^{\nu}\right), \quad d \rightarrow 0,
$$

for some $\nu>1$, where $\sigma_{\alpha}>0$ is given as

$$
\sigma_{\alpha}=2^{-1 / 2} \pi^{-1} m_{\alpha}^{3 / 2}\left|\left\langle\theta_{0}^{\alpha}, W_{\alpha}^{1 / 2}, \quad \phi_{1}^{\alpha}\right\rangle\right|^{2} .
$$

\section{§3. Proof of Theorem 1}

We keep the same notations as in the previous section. The proof of the theorem is long and is divided into several steps.

(1) We consider only $E, 0<E \ll 1$, small enough. For given self-adjoint operator $A$, we denote by $n(\lambda ; A)$ the number of eigenvalues greater than $\lambda$ of $A$. Let $U=\sum_{\alpha} U_{\alpha}$ and $W=\sum_{\alpha} W_{\alpha}$, where the summation $\Sigma_{\alpha}$ is taken over all three pairs $\alpha$. Define the Hamiltonian $K$ by

$$
K=H_{0}+U=H+W \text { on } L^{2}\left(R^{6}\right)
$$

and the bounded operator $M(E): L^{2}\left(R^{6}\right) \rightarrow L^{2}\left(R^{6}\right)$ by

$$
M(E)=(K+E)^{-1 / 2} W(K+E)^{-1 / 2}=\sum_{\alpha} M_{\alpha}(E)^{*} M_{\alpha}(E)
$$

with

$$
M_{\alpha}(E)=W_{\alpha}^{1 / 2}(K+E)^{-1 / 2} .
$$

Then the quantity $N(E)$ in question coincides with $n(1 ; M(E))$ by the BirmanSchwinger principle.

The next lemma is due to Sobolev [7]. For completeness, we here repeat the proof given there.

Lemma 3.1 Let

$$
\mathscr{L}^{2}=\sum \oplus L^{2}\left(R^{6}\right), \text { three summands. }
$$

Define the operator $\mathcal{M}(E): \mathscr{L}^{2} \rightarrow \mathscr{L}^{2}$ as

$$
\mu(E)=\left(\begin{array}{lll}
M_{\alpha}(E) M_{\alpha}(E)^{*} & M_{\alpha}(E) M_{\beta}(E)^{*} & M_{\alpha}(E) M_{\gamma}(E)^{*} \\
M_{\beta}(E) M_{\alpha}(E)^{*} & M_{\beta}(E) M_{\beta}(E)^{*} & M_{\beta}(E) M_{\gamma}(E)^{*} \\
M_{\gamma}(E) M_{\alpha}(E)^{*} & M_{\gamma}(E) M_{\beta}(E)^{*} & M_{\gamma}(E) M_{\gamma}(E)^{*}
\end{array}\right),
$$


where $\alpha, \beta$ and $\gamma$ denote different three pairs. Then one has

$$
N(E)=n(1 ; M(E)) .
$$

Proof. Let $\mathscr{L}(E): \mathscr{L}^{2} \rightarrow \mathscr{L}^{2}$ be defined as

$$
\mathscr{L}(E)=\frac{1}{\sqrt{3}}\left(\begin{array}{lll}
M_{\alpha}(E) & M_{\alpha}(E) & M_{\alpha}(E) \\
M_{\beta}(E) & M_{\beta}(E) & M_{\beta}(E) \\
M_{\gamma}(E) & M_{\gamma}(E) & M_{\gamma}(E)
\end{array}\right) .
$$

Then a simple calculation yields $\mathcal{M}(E)=\mathscr{L}(E) \mathscr{L}(E)^{*}$ and also we can easily see that the positive eigenvalues of $M(E)$ just coincide with those of $\mathscr{L}(E)^{*} \mathscr{L}(E)$. Hence the lemma follows at once.

(2) We denote by $\operatorname{Dia}\left\{B_{\alpha}, B_{\beta}, B_{\gamma}\right\}$ the $3 \times 3$ diagonal matrix with operators $B_{\alpha}, B_{\beta}$ and $B_{\gamma}$ as diagonal entries. Let $\mathcal{M}(E)$ be as in Lemma 3.1. The offdiagonal entries of $M(E)$ are all compact operators on $L^{2}\left(R^{6}\right)$ but the diagonal ones are not necessarily compact operators. Thus we look more carefully at the operator

$$
M_{\alpha}(E) M_{\alpha}(E)^{*}=W_{\alpha}^{1 / 2}(K+E)^{-1} W_{\alpha}^{1 / 2}
$$

in the diagonal entries of $\mathcal{M}(E)$.

Let $K_{\alpha}$ be defined by (2.4). We decompose the above operator into the sum $M_{\alpha}(E) M_{\alpha}(E)^{*}=M_{0 \alpha}(E)+L_{\alpha}(E)$, where $M_{0 \alpha}(E)=W_{\alpha}^{1 / 2}\left(K_{a}+E\right)^{-1} W_{\alpha}^{1 / 2}$ and

$$
L_{\alpha}(E)=W_{\alpha}^{1 / 2}\left((K+E)^{-1}-\left(K_{\alpha}+E\right)^{-1}\right) W_{\alpha}^{1 / 2},
$$

so that $\mathcal{M}(E)$ is represented as $\mathcal{M}(E)=\mathcal{M}_{0}(E)+\mathcal{M}_{1}(E)$ with

$$
M_{0}(E)=\operatorname{Dia}\left\{M_{0 \alpha}(E), M_{0 \beta}(E), M_{0 r}(E)\right\} .
$$

We note that $\mathcal{M}_{1}(E): \mathscr{L}^{2} \rightarrow \mathscr{L}^{2}$ is a compact operator.

We now introduce a positive smooth function $\omega(s), s>0$, such that

$$
\omega(s)=s \text { for } 0<s<1, \omega(s)=2 \text { for } s>2 .
$$

Let $T_{\alpha}$ be defined by (2.3) as an operator on $L^{2}\left(R^{3} ; d y_{\alpha}\right)$. We define

$$
\omega_{\alpha}(E)=\omega\left(\left(T_{\alpha}+E\right)^{1 / 2}\right) .
$$

This is considered as an operator acting on $L^{2}\left(R^{6}\right)$ as well as on $L^{2}\left(R^{3} ; d y_{\alpha}\right)$. We further define $A_{\alpha}(E): L^{2}\left(R^{6}\right) \rightarrow L^{2}\left(R^{6}\right)$ as

$$
A_{\alpha}(E)=\mathrm{Id}-M_{0 \alpha}(E)=\mathrm{Id}-W_{\alpha}^{1 / 2}\left(K_{\alpha}+E\right)^{-1} W_{a}^{1 / 2} .
$$


By Lemma 1.3 (see also Remark 1.4), we can find strictly positive smooth bounded functions $f(s)$ and $g(s), 0<c \leq f(s) \leq g(s)$, behaving like

$$
f(s)=1+o\left(s^{\nu}\right), g(s)=1+o\left(s^{\nu}\right), s \rightarrow 0,
$$

for some $\nu>0$ such that

$$
\begin{aligned}
& A_{\alpha}(E) \geq f_{\alpha}(E) \omega_{\alpha}(E) P_{1}^{\alpha}+c_{2} P_{2}^{\alpha}, \\
& A_{\alpha}(E) \leq g_{\alpha}(E) \omega_{\alpha}(E) P_{1}^{\alpha}+c_{2}^{\prime} P_{2}^{\alpha}
\end{aligned}
$$

for some positive constants $c_{2}$ and $c_{2}^{\prime}, 0<c_{2}<c_{2}^{\prime}$, where

$$
f_{\alpha}(E)=\sigma_{\alpha} f\left(\left(T_{\alpha}+E\right)^{1 / 2}\right), g_{\alpha}(E)=\sigma_{\alpha} g\left(\left(T_{\alpha}+E\right)^{1 / 2}\right)
$$

with $\sigma_{\alpha}>0$ given by (2.6), and the inequality relation is understood in the form sense. Denote by $F_{\alpha}(E)$ and $G_{\alpha}(E)$ the operators on the right side of (3.6) and (3.7), respectively, and define

$$
\begin{aligned}
& \mathscr{F}_{0}(E)=\operatorname{Dia}\left\{F_{a}(E), F_{\beta}(E), F_{\gamma}(E)\right\}, \\
& \mathscr{G}_{0}(E)=\operatorname{Dia}\left\{G_{a}(E), G_{\beta}(E), G_{\gamma}(E)\right\} .
\end{aligned}
$$

Then it follows from (3.6) and (3.7) that

$$
\mathscr{F}_{0}(E) \leq \mathrm{Id}-\mu_{0}(E), \leq \mathscr{G}_{0}(E)
$$

and hence we obtain from Lemma 3.1 that

$$
n\left(1 ; \mathscr{Q}^{g}(E)\right) \leq N(E) \leq n\left(1 ; \mathscr{Q}^{f}(E)\right),
$$

where

$$
\begin{aligned}
& \mathscr{Q}^{f}(E)=\mathscr{F}_{0}(E)^{-1 / 2} \mu_{1}(E) \mathscr{F}_{0}(E)^{-1 / 2}, \\
& \mathscr{Q}^{g}(E)=\mathscr{G}_{0}(E)^{-1 / 2} \mathcal{M}_{1}(E) \mathscr{G}_{0}(E)^{-1 / 2} .
\end{aligned}
$$

(3) We study the behavior as $E \rightarrow 0$ of Hilbert-Schmidt norm of the entry operators in $\mathscr{Q}^{f}(E)$ and $\mathscr{Q}^{g}(E)$. To do this, we here introduce the new notations. Let $B(E), 0<E \ll 1$, be a compact operator on $L^{2}\left(R^{6}\right)$. We say that $B(E)$ is of class $(H S)_{\varepsilon}$, if for any $\varepsilon>0$ small enough, $B(E)$ has a decomposition $B(E)=$ $B_{1}(E ; \varepsilon)+B_{2}(E ; \varepsilon)$ such that: (i) the Hilbert-Schmidt norm of $B_{1}(E ; \varepsilon)$ obeys the bound $\left\|B_{1}(E ; \varepsilon)\right\|_{H S} \leq C_{\varepsilon}$ for some $C_{\varepsilon}$ independent of $E$; (ii) the operator norm of $B_{2}(E ; \varepsilon)$ obeys the bound $\left\|B_{2}(E ; \varepsilon)\right\| \leq \varepsilon$. If the difference between two operators $B_{1}(E)$ and $B_{2}(E)$ is of class $(H S)_{\varepsilon}$, we denote this relation as $B_{1}(E) \sim$ $B_{2}(E)$. 
Let $K^{\alpha}$ be defined by $(2.4)$ as an operator acting on $L^{2}\left(R^{3} ; d x_{\alpha}\right)$. We denote by $R\left(d^{2} ; K^{\alpha}\right)$ the resolvent of $K^{\alpha} ; R\left(d^{2} ; K^{\alpha}\right)=\left(K^{\alpha}+d^{2}\right)^{-1}, d \geq 0$, and regard this as an operator acting on $L^{2}\left(R^{6}\right)$ as well as on $L^{2}\left(R^{3} ; d x_{\alpha}\right)$ in the discussion below. We further define $D_{0 \alpha}$ as

$$
D_{0 \alpha}=\mathrm{Id}-R\left(0 ; K^{\alpha}\right) U_{\alpha} .
$$

Lemma 3.2. Let $M_{\alpha}(E), L_{\alpha}(E)$ and $F_{\alpha}(E)$ be defined above. Then:

(1) $P_{2}^{\alpha} M_{\alpha}(E) M_{\beta}(E)^{*} P_{2}^{\beta} \in(H S)_{\varepsilon}, \quad \alpha \neq \beta$.

(2) $P_{1}^{\alpha} \omega_{\alpha}(E)^{-1 / 2} M_{\alpha}(E) M_{\beta}(E)^{*} P_{2}^{\beta} \in(H S)_{\varepsilon}, \quad \alpha \neq \beta$.

(3) $F_{a}(E)^{-1 / 2} L_{\alpha}(E) F_{\alpha}(E)^{-1 / 2} \in(H S)_{\varepsilon}$.

We proceed with the argument, accepting this lemma as proved. We now denote by $Q_{\alpha \beta}^{f}(E)$ the entry operators of $\mathscr{Q}^{f}(E)$. By Lemma 3.2, the diagonal entry operators $Q_{\alpha \alpha}^{f}(E)$ are all of class $(H S)_{\varepsilon}$ and the off-diagonal ones $Q_{\alpha \beta}^{f}(E), \alpha \neq \beta$, take the form

$$
Q_{\alpha \beta}^{f}(E) \sim P_{1}^{\alpha} W_{\alpha}^{1 / 2}\left(f_{\alpha}(E) \omega_{\alpha}(E)\right)^{-1 / 2}(K+E)^{-1}\left(f_{\beta}(E) \omega_{\beta}(E)\right)^{-1 / 2} W_{\beta}^{1 / 2} P_{1}^{\beta} .
$$

A similar relation holds for the entry operators $Q_{\alpha \beta}^{g}(E)$ of $\mathscr{Q}^{g}(E)$ with $f_{\alpha}(E)$ replaced by $g_{\alpha}(E)$. We further continue to analyse the operator on the right side of the relation above.

Lemma 3.3 Let $D_{0 \alpha}$ be defined by (3.10). Define $T_{\alpha \beta}(E): L^{2}\left(R^{6}\right) \rightarrow L^{2}\left(R^{6}\right)$ by

$$
T_{\alpha \beta}(E)=\left(\sigma_{\alpha} \sigma_{\beta}\right)^{-1 / 2} P_{1}^{\alpha} W_{\alpha}^{1 / 2} D_{0 \alpha} \Gamma_{\alpha \beta}(E) D_{0 \beta}^{*} W_{\beta}^{1 / 2} P_{1}^{\beta},
$$

where $\sigma_{\alpha}$ is given by (2.6) and

$$
\Gamma_{\alpha \beta}(E)=\omega_{\alpha}(E)^{-1 / 2}\left(H_{0}+E\right)^{-1} \omega_{\beta}(E)^{-1 / 2} .
$$

Then one has

$$
Q_{\alpha \beta}^{f}(E) \sim T_{\alpha \beta}(E), \quad \alpha \neq \beta .
$$

A similar relation holds for the entry operators $Q_{\alpha \beta}^{g}(E)$ of $\mathscr{Q}^{g}(E)$.

The two lemmas above are proved in section 5 .

(4) Let $\Psi_{\alpha}: L^{2}\left(R^{3} ; d y_{\alpha}\right) \rightarrow L^{2}\left(R^{3} ; d q_{\alpha}\right)$ be the Fourier transformation in $y_{\alpha}$. We define 


$$
\Lambda_{\alpha \beta}(E)=\Psi_{\alpha} T_{\alpha \beta}(E) \Psi_{\beta}^{*}: L^{2}\left(R^{6} ; d x_{\beta} d q_{\beta}\right) \rightarrow L^{2}\left(R^{6} ; d x_{\alpha} d q_{\alpha}\right)
$$

for $T_{\alpha \beta}(E)$ in Lemma 3.3. The aim in this step is to study the integral kernel of $\Lambda_{\alpha \beta}(E)$.

We begin by recalling the following notations: $\theta_{0}^{\alpha}=\theta_{0}^{\alpha}\left(x_{\alpha}\right)$ is the generalized eigenfunction at zero energy of $K^{\alpha} ; \phi_{1}^{\alpha} \in L^{2}\left(R^{3} ; d x_{\alpha}\right)$ is the normalized function spanning the range of $P_{1}^{\alpha}$ (one-dimensional space). Let $D_{0 \alpha}$ be defined by (3.10). Then the generalized eigenfunction $\theta_{0}^{\alpha}$ is given as

$$
\theta_{0}^{\alpha}=D_{0 \alpha} 1=1-R\left(0 ; K^{\alpha}\right) U_{\alpha} 1
$$

and satisfies

$$
\left\langle\theta_{0}^{\alpha}, W_{\alpha}^{1 / 2} \phi_{1}^{\alpha}\right\rangle=\left\langle 1, D_{0 \alpha}^{*} W_{\alpha}^{1 / 2} \phi_{1}^{\alpha}\right\rangle \neq 0,
$$

where $\langle$,$\rangle denotes the scalar product in L^{2}\left(R^{3} ; d x_{\alpha}\right)$. We may assume that this quantity is positive, so that it is represented as

$$
\left\langle\theta_{0}^{\alpha}, W_{\alpha}^{1 / 2} \phi_{0}^{\alpha}\right\rangle=2^{1 / 4} \pi^{1 / 2} m_{\alpha}^{-3 / 4} \sigma_{\alpha}^{1 / 2}
$$

for $\sigma_{\alpha}$ defined by (2.6). We denote by $\Phi_{\alpha}: L^{2}\left(R^{3} ; d x_{\alpha}\right) \rightarrow L^{2}\left(R^{3} ; d p_{\alpha}\right)$ the Fourier transformation in $x_{\alpha}$ and define the function $\varphi_{1}^{\alpha}\left(p_{\alpha}\right) \in L^{2}\left(R^{3} ; d p_{\alpha}\right)$ as $\varphi_{1}^{\alpha}=\left(\Phi_{\alpha} D_{0 \alpha}^{*} W_{\alpha}^{1 / 2} \phi_{1}^{\alpha}\right)\left(p_{\alpha}\right)$;

$$
\varphi_{1}^{\alpha}\left(p_{\alpha}\right)=(2 \pi)^{-3 / 2} \int \exp \left(-i p_{\alpha} \cdot x_{\alpha}\right)\left(D_{0 \alpha}^{*} W_{\alpha}^{1 / 2} \phi_{1}^{\alpha}\right)\left(x_{\alpha}\right) d x_{\alpha} .
$$

Since $\phi_{1}^{\alpha} \in$ Range $P_{1}^{\alpha}$ satisfies the relation

$$
\phi_{1}^{\alpha}=W_{\alpha}^{1 / 2} R\left(0 ; K^{\alpha}\right) W_{\alpha}^{1 / 2} \phi_{1}^{\alpha}
$$

and since $0 \leq U_{\alpha} W_{\alpha}^{-1 / 2} \leq \sqrt{2} U_{\alpha}^{1 / 2}$ by (1.3), it follows from Lemma 1.2 that

$$
\left\langle x_{\alpha}\right\rangle^{\nu} D_{0 \alpha}^{*} W_{\alpha}^{1 / 2} \phi_{1}^{\alpha}=\left\langle x_{\alpha}\right\rangle^{\nu}\left(W_{\alpha}^{1 / 2}-U_{\alpha} W_{\alpha}^{-1 / 2}\right) \phi_{1}^{\alpha}
$$

is integrable for $\nu, 0<\nu<\rho-2$, and hence we have that $\varphi_{1}^{\alpha}\left(p_{\alpha}\right)$ behaves like

$$
\varphi_{1}^{\alpha}\left(p_{\alpha}\right)=2^{-5 / 4} \pi^{-1} m_{\alpha}^{-3 / 4} \sigma_{\alpha}^{1 / 2}+O\left(\left|p_{\alpha}\right|^{\nu}\right), \quad\left|p_{\alpha}\right| \rightarrow 0
$$

for $\nu>0$ as above.

We now return to the operator $\Lambda_{\alpha \beta}(E): L^{2}\left(R^{6} ; d x_{\beta} d q_{\beta}\right) \rightarrow L^{2}\left(R^{6}\right.$; $\left.d x_{\alpha} d q_{\alpha}\right)$ defined above. As is easily seen, this operator has an integral kernel of the form

$$
\left(\sigma_{\alpha} \sigma_{\beta}\right)^{-1 / 2} \phi_{1}^{\alpha}\left(x_{\alpha}\right) \varphi_{1}^{-\alpha}\left(p_{\alpha}\right) G\left(q_{\alpha}, q_{\beta} ; E\right) \varphi_{1}^{\beta}\left(p_{\beta}\right) \phi_{1}^{\beta}\left(x_{\beta}\right)
$$


where $p_{\alpha}$ and $p_{\beta}$ are represented as in (2.2) in terms of the coordinates $\left(q_{\alpha}, q_{\beta}\right)$ and $G\left(q_{\alpha}, q_{\beta}: E\right)$ is defined by

$$
\omega\left(\left(\left|q_{\alpha}\right|^{2} / 2 n_{\alpha}+E\right)^{1 / 2}\right)^{-1 / 2}\left(H_{0}\left(q_{\alpha}, q_{\beta}\right)+E\right)^{-1} \omega\left(\left(\left|q_{\beta}\right|^{2} / 2 n_{\beta}+E\right)^{1 / 2}\right)^{-1 / 2}
$$

with the symbol representation $H_{0}\left(q_{\alpha}, q_{\beta}\right)$ for the free Hamiltonian $H_{0}$ in the coordinate system $\left(q_{\alpha}, q_{\beta}\right)$.

(5) Let $\chi(x), x \in R^{3}$, be the characteristic function of the unit ball $B_{1}$ in $R^{3}$. We set

$$
\zeta_{\alpha}\left(q_{\alpha} ; E\right)=\chi\left(q_{\alpha}\right)\left(\left|q_{\alpha}\right|^{2} / 2 n_{\alpha}+E\right)^{-1 / 4}
$$

and denote by $\Pi_{\alpha \beta}(E): L^{2}\left(R^{6} ; d x_{\beta} d q_{\beta}\right) \rightarrow L^{2}\left(R^{6} ; d x_{\alpha} d q_{\alpha}\right), a \neq \beta$, the integral operator with the kernel $\phi_{1}^{\alpha}\left(x_{\alpha}\right) J_{\alpha \beta}\left(q_{\alpha}, q_{\beta} ; E\right) \phi_{1}^{\beta}\left(x_{\beta}\right)$, where $J_{\alpha \beta}\left(q_{\alpha}, q_{\beta} ; E\right)$ is defined by

$$
J_{\alpha \beta}\left(q_{\alpha}, q_{\beta} ; E\right)=\tau_{\alpha \beta} \zeta_{\alpha}\left(q_{\alpha} ; E\right)\left(H_{0}\left(q_{\alpha}, q_{\beta}\right)+E\right)^{-1} \zeta_{\beta}\left(q_{\beta} ; E\right)
$$

with

$$
\tau_{\alpha \beta}=2^{-5 / 2} \pi^{-2}\left(m_{\alpha} m_{\beta}\right)^{-3 / 4} .
$$

We further define $S_{\alpha \beta}(E): L^{2}\left(R^{6}\right) \rightarrow L^{2}\left(R^{6}\right)$ by $S_{\alpha \beta}(E)=\Psi_{\alpha}^{*} \Pi_{\alpha \beta}(E) \Psi_{\beta}, \alpha \neq \beta$, and the self-adjoint operator $\mathscr{S}(E): \mathscr{L}^{2} \rightarrow \mathscr{L}^{2}, \mathscr{L}^{2}$ being as in Lemma 3.1 , by

$$
\mathscr{S}(E)=\left(\begin{array}{ccc}
0 & S_{\alpha \beta}(E) & S_{\alpha \gamma}(E) \\
S_{\beta \alpha}(E) & 0 & S_{\beta \gamma}(E) \\
S_{\gamma \alpha}(E) & S_{\gamma \beta}(E) & 0
\end{array}\right), \quad S_{\beta \alpha}(E)=S_{\alpha \beta}(E)^{*} .
$$

Then it follows from (3.11) that the Hilbert-Schmidt norm of the difference

$$
T_{\alpha \beta}(E)-S_{\alpha \beta}(E)=\Psi_{\alpha}^{*}\left(\Lambda_{\alpha \beta}(E)-\Pi_{\alpha \beta}(E)\right) \Psi_{\beta}
$$

is bounded uniformly in $E$ and hence we have

$$
Q_{\alpha \beta}^{f}(E) \sim S_{\alpha \beta}(E) \text { and } Q_{\alpha \beta}^{g}(E) \sim S_{\alpha \beta}(E), \quad \alpha \neq \beta,
$$

by Lemma 3.3. This, together with (3.9), yields

$$
n((1+\varepsilon) ; \oiint(E))-C_{\varepsilon} \leq N(E) \leq n((1-\varepsilon) ; \oiint(E))+C_{\varepsilon}
$$

for any $\varepsilon>0$ small enough, where $C_{\varepsilon}>0$ is independent of $E$. This relation is obtained by use of the Weyl inequality

$$
n\left(\lambda_{1}+\lambda_{2} ; A_{1}+A_{2}\right) \leq n\left(\lambda_{1} ; A_{1}\right)+n\left(\lambda_{2} ; A_{2}\right)
$$


for the sum of compact operators $A_{1}$ and $A_{2}$.

(6) The proof of the theorem is completed in this step. Let

$$
\mathscr{L}^{2}\left(B_{1}\right)=\sum_{\alpha} \oplus L^{2}\left(B_{1} ; d q_{\alpha}\right), \text { three summands. }
$$

We denote by $J_{\alpha \beta}(E): L^{2}\left(B_{1} ; d q_{\beta}\right) \rightarrow L^{2}\left(B_{1} ; d q_{\alpha}\right)$ the integral operator with the kernel $J_{\alpha \beta}\left(q_{\alpha}, q_{\beta} ; E\right)$ defined by (3.12), and define the operator $\mathscr{J}_{0}(E): \mathscr{L}^{2}\left(B_{1}\right) \rightarrow$ $\mathscr{L}^{2}\left(B_{1}\right)$ as

$$
g_{0}(E)=\left(\begin{array}{ccc}
0 & J_{\alpha \beta}(E) & J_{\alpha \gamma}(E) \\
J_{\beta \alpha}(E) & 0 & J_{\beta \gamma}(E) \\
J_{\gamma \alpha}(E) & J_{\gamma \beta}(E) & 0
\end{array}\right) .
$$

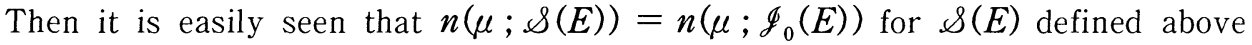
and hence we have

$$
n\left((1+\varepsilon) ; \mathscr{g}_{0}(E)\right)-C_{\varepsilon} \leq N(E) \leq n\left((1-\varepsilon) ; \mathscr{g}_{0}(E)\right)+C_{\varepsilon} .
$$

The eigenvalue asymptotics for the integral operator $\mathscr{g}_{0}(E)$ has been in detail studied in Sobolev [7] by employing an argument used in the calculation of the canonical distribution of Toeplitz operators. We here summarize the results obtained there.

Lemma 3.4. Let $n\left(\mu ; \mathscr{g}_{0}(E)\right)$ be as above. Then:

(1) There exists a limit

$$
\Theta_{0}(\mu)=\lim _{E \rightarrow 0} n\left(\mu ; \mathscr{g}_{0}(E)\right) /|\log E|
$$

as a continuous function of $\mu>0$.

(2) The constant $C_{0}=\Theta_{0}(1)$ depends only on the ratios between the masses of three particles under consideration and obeys the lower bound

$$
C_{0}>\log 2 / 2 \pi^{2}>0 .
$$

(3) In the special case with identical masses, $C_{0}$ is explicitly calculated as $C_{0}=$ $\lambda / 2 \pi$ with the unique positive root $\lambda>0$ of the equation

$$
\lambda=2^{3} \cdot 3^{-1 / 2}(\sinh \pi \lambda / 6) /(\cosh \pi \lambda / 2) .
$$

This lemma, together with relation (3.13), completes the proof of the theorem. 


\section{\$4. Proof of Lemma 1.3}

The three lemmas (Lemmas 1.3, 3.2 and 3.3) remain unproved. We prove Lemma 1.3 in the present section and Lemma 3.2 and 3.3 in the next one.

We keep the same notations as in section 1 and prove successively a series of lemmas below, which completes the proof of Lemma 1.3.

Lemma 4.1. $\quad c_{\varepsilon} \operatorname{Id} \leq A(d) \leq c_{\varepsilon}^{\prime}$ Id for $d \geq \varepsilon>0$.

Lemma 4.2. $A_{22}(d) \in \mathrm{Op}\left(d^{0}\right)$ and $A_{22}(d) \geq c_{2} P_{2}$ for some $c_{2}>0$.

Lemma 4.3. $A_{12}(d) \in \mathrm{Op}\left(d^{\nu}\right)$ for some $\nu>1 / 2$.

Lemma 4.4. $A_{11}(d)=\sigma_{1} d P_{1}+\mathrm{Op}\left(d^{\nu}\right)$ for some $\nu>1$.

Proof of Lemma 4.1. The upper bound is obvious. The lower bound is proved by contradiction. Assume that such a positive constant $c_{\varepsilon}$ does not exist. Then the compact operator $W_{0}^{1 / 2} R\left(d^{2} ; S\right) W_{0}^{1 / 2}$ has an eigenvalue greater than one or equal to one for some $d \geq \varepsilon$. This implies that $T$ has a negative eigenvalue and contradicts the assumption (H.1). Hence the lemma is proved.

4.1. To prove Lemmas $4.2 \sim 4.4$, we use the low energy expansion of two-body resolvents. We here make a brief review on this result, following the idea due to [2].

Let $T_{0}=-\Delta$ be the free Hamiltonian on $L^{2}\left(R_{x}^{3}\right)$. We denote by $R_{0}\left(d^{2}\right)=$ $R\left(d^{2} ; T_{0}\right), d \geq 0$, the resolvent of $T_{0} ; R_{0}\left(d^{2}\right)=\left(T_{0}+d^{2}\right)^{-1}$. The operator $R_{0}\left(d^{2}\right)$ is an integral operator with the kernel

$$
R_{0}\left(d^{2}\right):(1 / 4 \pi) \exp (-d|x-y|) /|x-y|
$$

and admits the formal expansion

$$
R_{0}\left(d^{2}\right)=\sum_{j=0}^{\infty}(-1)^{j} d^{j} G_{j}
$$

where

$$
G_{j}:(1 / 4 \pi j !)|x-y|^{j-1}, \quad j=0,1,2, \ldots
$$

We denote by $\mathscr{B}\left(s ; s^{\prime}\right)$ the class of bounded operators from the weighted $L^{2}$ space 
$L_{s}^{2}=L_{s}^{2}\left(R_{x}^{3}\right)$ into $L_{s^{\prime}}^{2}$. The operators $R_{0}\left(d^{2}\right), G_{0}$ and $G_{1}$ can be easily proved to have the following properties.

Lemma 4.5. (1) $R_{0}\left(d^{2}\right) \in \mathscr{B}\left(s ;-s^{\prime}\right)$ for $s, s^{\prime}>1 / 2, s+s^{\prime}>2$, and the operator norm in $\mathscr{B}\left(s ;-s^{\prime}\right)$ is bounded uniformly in $d \geq 0$.

(2) $G_{0} \in \mathscr{B}\left(s ; s^{\prime}\right)$ for the same pair $\left(s, s^{\prime}\right)$ as above.

(3) $G_{1} \in \mathscr{B}(s,-s)$ for $s>3 / 2$.

Let $S=-\Delta+U_{0}$ be defined by (1.4). Since $U_{0}$ in non-negative, the resolvent $R\left(d^{2} ; S\right), d \geq 0$, has the same property as $R_{0}\left(d^{2}\right)$.

Lemma 4.6. $R\left(d^{2} ; S\right) \in \mathscr{B}\left(s ;-s^{\prime}\right)$ for $s, s^{\prime}>1 / 2, s+s^{\prime}>2$, and the operator norm in $\mathscr{B}\left(s ;-s^{\prime}\right)$ is bounded uniformly in $d \geq 0$.

We define $Z(d)$ as

$$
Z(d)=R_{0}\left(d^{2}\right)-G_{0}
$$

and $D_{0}$ as

$$
D_{0}=\left(\mathrm{Id}+G_{0} U_{0}\right)^{-1}=\mathrm{Id}-R(0 ; S) U_{0} .
$$

Then $R\left(d^{2} ; S\right)$ is represented as

$$
R\left(d^{2} ; S\right)=\left(\operatorname{Id}+R_{0}\left(d^{2}\right) U_{0}\right)^{-1} R_{0}\left(d^{2}\right)=\left(\operatorname{Id}+D_{0} Z(d) U_{0}\right)^{-1} D_{0} R_{0}\left(d^{2}\right) .
$$

As the Neumann series, the inverse above is expanded as

$$
\sum_{j=0}^{n-1}(-1)^{j}\left(D_{0} Z(d) U_{0}\right)^{j}+(-1)^{n}\left(\operatorname{Id}+D_{0} Z(d) U_{0}\right)^{-1}\left(D_{0} Z(d) U_{0}\right)^{n} .
$$

Since

$$
\left(\mathrm{Id}+D_{0} Z(d) U_{0}\right)^{-1} D_{0}=\left(\operatorname{Id}+R_{0}\left(d^{2}\right) U_{0}\right)^{-1}=\operatorname{Id}-R\left(d^{2} ; S\right) U_{0},
$$

we obtain that $R\left(d^{2} ; S\right)$ is expanded as

$$
\begin{aligned}
R\left(d^{2} ; S\right)= & \sum_{j=0}^{n-1}(-1)^{j}\left(D_{0} Z(d) U_{0}\right)^{j} D_{0} R_{0}\left(d^{2}\right) \\
& +(-1)^{n}\left(\operatorname{Id}-R\left(d^{2} ; S\right) U_{0}\right)\left(Z(d) U_{0} D_{0}\right)^{n} R_{0}\left(d^{2}\right) .
\end{aligned}
$$

This expansion formula with $1 \leq n \leq 2$ is used in the proof of Lemmas $4.2 \sim 4.4$. 
4.2. We here prove Lemmas 4.2 and 4.3. In the discussion below, $X_{s}$ denote the multiplication by $\langle x\rangle^{s}$. The following three lemmas are obtained as an immediate consequence of Lemmas 4.5 and 4.6 .

Lemma 4.7. Let $D_{0}$ be defined by (4.2). Then $D_{0} \in \mathscr{B}(-s ;-s)$ for any $s$. $1 / 2<s<\rho-1 / 2$.

Lemma 4.8.

$$
P_{2} W_{0}^{1 / 2}\left(\mathrm{Id}-R\left(d^{2} ; S\right) U_{0}\right) X_{\rho / 2} \in \mathrm{Op}\left(d^{0}\right)
$$

and, in particular, $P_{2} W_{0}^{1 / 2} D_{0} \in \mathscr{B}(-\rho / 2 ; 0)$.

Lemma 4.9.

$$
X_{\rho / 2} U_{0} D_{0} R_{0}\left(d^{2}\right) W_{0}^{1 / 2} P_{2} \in \mathrm{Op}\left(d^{0}\right) .
$$

Let $Z(d)$ be defined by (4.1). The kernel $Z(x, y ; d)$ of $Z(d)$ obeys the bound

$$
|Z(x, y ; d)| \leq C_{\sigma} d^{\sigma}|x-y|^{\sigma-1}
$$

for any $\sigma, 0 \leq \sigma \leq 1$. Hence we can easily prove the following

Lemma 4.10. Let $\sigma$ be such that $0 \leq \sigma \leq 1$. The operator $Z(d)$ has the following properties:

(1) $Z(d) \in \mathscr{B}(s ;-s)$ for $s>1+\sigma / 2$ and $X_{-s} Z(d) X_{-s} \in \mathrm{Op}\left(d^{\sigma}\right)$.

(2) $Z(d) \in \mathscr{B}\left(s ;-s^{\prime}\right)$ for $s>1 / 2+\sigma, s^{\prime}>3 / 2$ or for $s>3 / 2, s^{\prime}>1 / 2$ $+\sigma$, and $X_{-s^{\prime}} Z(d) X_{-s} \in \mathrm{Op}\left(d^{\sigma}\right)$.

Proof of Lemma 4.2. By the expansion formula (4.3) with $n=1, A_{22}(d)$ is represented as the sum of two operators $J_{0}(d)$ and $J_{1}(d)$, where

$$
\begin{aligned}
& J_{0}(d)=P_{2}\left(\mathrm{Id}-W_{0}^{1 / 2} D_{0} R_{0}\left(d^{2}\right) W_{0}^{1 / 2}\right) P_{2}, \\
& J_{1}(d)=P_{2} W_{0}^{1 / 2}\left(\operatorname{Id}-R\left(d^{2} ; S\right) U_{0}\right) Z(d) U_{0} D_{0} R_{0}\left(d^{2}\right) W_{0}^{1 / 2} P_{2} .
\end{aligned}
$$

We apply Lemma 4.10 to the operator $Z(d)$ in $J_{1}(d)$, considering this as an operator of class $\mathscr{B}(\rho / 2 ;-\rho / 2), \rho / 2>1$. Then it follows from Lemmas 4.8 and 4.9 that $J_{1}(d) \in \mathrm{Op}\left(d^{\nu}\right)$ for some $\nu>0$. We write $D_{0} R_{0}\left(d^{2}\right)$ as

$$
D_{0} R_{0}\left(d^{2}\right)=D_{0}\left(G_{0}+Z(d)\right)=R(0 ; S)+D_{0} Z(d) .
$$

Then the same argument as above shows that $J_{0}(d)=P_{2} A(0) P_{2}+\mathrm{Op}\left(d^{2}\right)$ for 
some $\nu>0$, so that we have

$$
A_{22}(d)=P_{2} A(0) P_{2}+\mathrm{Op}\left(d^{\nu}\right) .
$$

By assumption (H.1), $P_{2} A(0) P_{2} \geq c_{2} P_{2}$ for some $c_{2}>0$. This proves the lemma.

Next we prove Lemma 4.3. Since $P_{1} \in \mathscr{B}(-s ; s)$ for $s, 1 / 2<s<$ $(\rho-1) / 2$, by Lemma 1.2 , the following lemma can be easily verified.

LEMma 4.11.

$$
P_{1} W_{0}^{1 / 2}\left(\mathrm{Id}-R\left(d^{2} ; S\right) U_{0}\right) X_{s} \in \mathrm{Op}\left(d^{0}\right)
$$

for any $s, 1 / 2<s<\rho-1 / 2$, and, in particular, $P_{1} W_{0}^{1 / 2} D_{0} \in \mathscr{B}(-s ; 0)$.

Proof of Lemma 4.3. We again use the expansion formula (4.3) with $n=1$. If we make use of the orthogonal property of projections $P_{1}$ and $P_{2}, A_{12}(d)$ is represented as the sum of two operators $J_{0}(d)$ and $J_{1}(d)$, where

$$
\begin{aligned}
& J_{0}(d)=-P_{1} W_{0}^{1 / 2} D_{0} R_{0}\left(d^{2}\right) W_{0}^{1 / 2} P_{2}, \\
& J_{1}(d)=P_{1} W_{0}^{1 / 2}\left(\operatorname{Id}-R\left(d^{2} ; S\right) U_{0}\right) Z(d) U_{0} D_{0} R_{0}\left(d^{2}\right) W_{0}^{1 / 2} P_{2} .
\end{aligned}
$$

We apply Lemma 4.10 to the operator $Z(d)$ in $J_{1}(d)$. To do this, we take $s$ as $3 / 2$ $<s<\rho-1 / 2$ and consider this as an operator of class $\mathscr{B}(\rho / 2 ;-s)$, so that $Z(d)$ obeys the bound $O\left(d^{\nu}\right)$ for some $\nu>1 / 2$ in this operator class. Hence we obtain from Lemmas 4.9 and 4.11 that $J_{1}(d) \in \mathrm{Op}\left(d^{\nu}\right)$. Similarly we have

$$
J_{0}(d)=-P_{1} W_{0}^{1 / 2} R(0 ; S) W_{0}^{1 / 2} P_{2}+\mathrm{Op}\left(d^{\nu}\right) .
$$

However, the operator on the right side vanishes by the orthogonality of $P_{1}$ and $P_{2}$. This proves the lemma.

4.3. We end this section by proving Lemma 4.4. The next lemma is easy to verify. In fact, it follows from Lemmas 1.2 and 4.7 at once.

LeMma 4.12.

$$
X_{s} U_{0} D_{0} R_{0}\left(d^{2}\right) W_{0}^{1 / 2} P_{1} \in \mathrm{Op}\left(d^{0}\right)
$$

for any $s, 3 / 2<s<\rho-1 / 2$.

Let $G_{1}$ be as in 4.1 . Recall that this is the integral operator with kernel $1 / 4 \pi$ 
and acts as

$$
G_{1} u=(1 / 4 \pi)\langle u, 1\rangle 1
$$

as an operator from $L_{s}^{2}$ into $L_{-s}^{2}$ for $s>3 / 2$. We define $Z_{1}(d), 0<d \ll 1$, as

$$
Z_{1}(d)=R_{0}\left(d_{2}\right)-G_{0}+d G_{1}=Z(d)+d G_{1} .
$$

The kernel $Z_{1}(x, y ; d)$ of $Z_{1}(d)$ obeys the bound

$$
\left|Z_{1}(x, y ; d)\right| \leq C_{\sigma} d^{\sigma}|x-y|^{\sigma-1}
$$

for any $\sigma$ with $1 \leq \sigma \leq 2$. Thus we can easily prove the following

Lemma 4.13. Let $\sigma$ be such that $1 \leq \sigma \leq 2$. Then $Z_{1}(d) \in \mathscr{B}(s ;-s)$ for $s>1 / 2+\sigma$, and $X_{-s} Z_{1}(d) X_{-s} \in \mathrm{Op}\left(d^{\sigma}\right)$.

Proof of Lemma 4.4. We use the expansion formula (4.3) with $n=2$. Then the operator $A_{11}(d)$ is decomposed into the sum of three operators $J_{j}(d)$, $0 \leq j \leq 2$, where

$$
\begin{aligned}
& J_{0}(d)=P_{1}\left(\mathrm{Id}-W_{0}^{1 / 2} D_{0} R_{0}\left(d^{2}\right) W_{0}^{1 / 2}\right) P_{1}, \\
& J_{1}(d)=P_{1} W_{0}^{1 / 2} D_{0} Z(d) U_{0} D_{0} R_{0}\left(d^{2}\right) W_{0}^{1 / 2} P_{1}, \\
& J_{2}(d)=-P_{1} W_{0}^{1 / 2}\left(\mathrm{Id}-R\left(d^{2} ; S\right) U_{0}\right)\left(Z(d) U_{0} D_{0}\right)^{2} R_{0}\left(d^{2}\right) W_{0}^{1 / 2} P_{1} .
\end{aligned}
$$

We first consider the operator $J_{2}(d)$. This has two operators $Z(d)$. We apply Lemma 4.10 to control these operators. To this end, we take $s$ as $3 / 2<s<\rho-1 / 2$ and consider $Z(d)$ on the left side as an operator of class $\mathscr{B}(\rho / 2 ;-s)$ and $Z(d)$ on the right side as an operator of class $\mathscr{B}(s ;-\rho / 2)$, so that both the operators obey the bound $O\left(d^{\nu / 2}\right)$ for some $\nu>1$ in the classes under consideration. Since $U_{0} D_{0} \in \mathscr{B}(-\rho / 2 ; \rho / 2)$ by Lemma 4.7 , it follows from Lemmas 4.11 and 4.12 that $J_{2}(d) \in \mathrm{Op}\left(d^{\nu}\right)$. To evaluate $J_{1}(d)$, we rewrite it as

$$
J_{1}(d)=P_{1} W_{0}^{1 / 2} D_{0}\left(-d G_{1}+Z_{1}(d)\right) U_{0} D_{0} R_{0}\left(d^{2}\right) W_{0}^{1 / 2} P_{1}
$$

and apply Lemma 4.13 to $Z_{1}(d)$. We again take $s$ as $3 / 2<s<\rho-1 / 2$ and consider $Z_{1}(d)$ as an operator of class $\mathscr{B}(s ;-s)$. Then we have by Lemmas 4.11 $\sim 4.13$ that

$$
J_{1}(d)=-d P_{1} W_{0}^{1 / 2} D_{0} G_{1} U_{0} D_{0}\left(G_{0}+Z(d)\right) W_{0}^{1 / 2} P_{1}+\mathrm{Op}\left(d^{\nu}\right)
$$

for some $\nu>1$. We further apply Lemma 4.10 to $Z(d)$ above, considering this as an operator of class $\mathscr{B}(s ;-(\rho-s))$. Since $U_{0} D_{0} \in \mathscr{B}(-(\rho-s) ; s)$ and 
$G_{1} \in \mathscr{B}(s ;-s)$, we have

$$
J_{1}(d)=-d P_{1} W_{0}^{1 / 2} D_{0} G_{1} U_{0} D_{0} G_{0} W_{0}^{1 / 2} P_{1}+\mathrm{Op}\left(d^{\nu}\right) .
$$

If we take account of the relation

$$
P_{1}\left(\mathrm{Id}-W_{0}^{1 / 2} D_{0} G_{0} W_{0}^{1 / 2}\right) P_{1}=P_{1} A(0) P_{1}=0,
$$

we can similarly show that $J_{0}(d)$ is represented in the form

$$
J_{0}(d)=d P_{1} W_{0}^{1 / 2} D_{0} G_{1} W_{0}^{1 / 2} P_{1}+\mathrm{Op}\left(d^{\nu}\right)
$$

with another $\nu>1$.

We now use the relation

$$
U_{0} D_{0} G_{0}=U_{0}\left(\mathrm{Id}+G_{0} U_{0}\right)^{-1} G_{0}=\left(\mathrm{Id}+U_{0} G_{0}\right)^{-1} U_{0} G_{0}
$$

to obtain that

$$
D_{0} G_{1}\left(\mathrm{Id}-U_{0} D_{0} G_{0}\right)=D_{0} G_{1}\left(\mathrm{Id}+U_{0} G_{0}\right)^{-1}=D_{0} G_{1} D_{0}^{*},
$$

so that

$$
A_{11}(d)=d P_{1} W_{0}^{1 / 2} D_{0} G_{1} D_{0}^{*} W_{0}^{1 / 2} P_{1}+\mathrm{Op}\left(d^{\nu}\right) .
$$

The generalized eigenfunction $\theta_{0}$ at zero energy of $S$ is given as

$$
\theta_{0}=D_{0} 1=1-R(0 ; S) U_{0} 1
$$

and satisfies $\left\langle\theta_{0}, W_{0}^{1 / 2} \phi_{1}\right\rangle \neq 0$ for the normalized function $\phi_{1} \in L^{2}$ spanning $\Sigma_{1}=$ Range $P_{1}, \Sigma_{1}$ being of one dimension. Thus, by (4.4), we have

$$
P_{1} W_{0}^{1 / 2} D_{0} G_{1} D_{0}^{*} W_{0}^{1 / 2} P_{1}=\sigma_{1} P_{1}
$$

for $\sigma_{1}$ as in Lemma 1.3. This completes the proof.

\section{\$5. Proof of Lemmas 3.2 and 3.3}

In this section we prove Lemmas 3.2 and 3.3. Throughout the section, we use the following notations: $X_{s}^{\alpha}$ denotes the multiplication operator by $\left\langle x_{\alpha}\right\rangle^{s}$; Op $\left(E_{0}\right)$ denotes the class of bounded operators on $L^{2}\left(R^{6}\right)$ uniformly in $E, 0<E$ $\ll 1$, small enough and various operators of class $O p\left(E^{0}\right)$ are denoted by the same symbol $r_{0}(E)$.

5.1. The first half of this section is devoted to preparing a series of auxiliary lemmas which are required to prove the lemmas above. 
Lemma 5.1. Let $0 \leq \nu<3 / 2$. Assume that $\alpha \neq \beta$. Then

$$
X_{-s}^{\beta} \omega_{\alpha}(E)^{-\nu} \in \mathrm{Op}\left(E^{0}\right) \text { for any } s>\nu .
$$

Remark. As an immediate consequence of the lemma, it follows that $\omega_{\alpha}(E)^{-\nu} X_{-s}^{\beta} \in \mathrm{Op}\left(E^{0}\right)$. Throughout the discussion below, such simple consequences are used without further references.

Proof. Let $\theta$ be the characteristic function of the interval $[0,1]$. To prove the lemma, it suffices to show that $\theta\left(T_{\alpha}\right) \omega_{\alpha}(E)^{-\nu}$ has the property above. By the assumption $\alpha \neq \beta$, the coordinates $x_{\beta}$ are described as $x_{\beta}=\zeta_{\beta \alpha} x_{\alpha}+\kappa_{\beta \alpha} y_{\alpha}$ with $\kappa_{\beta \alpha} \neq 0$. We consider $X_{-s}^{\beta} \theta\left(T_{\alpha}\right) \omega_{\alpha}(E)^{-\nu}$ as an operator acting on $L^{2}\left(R^{3} ; d y_{\alpha}\right), x_{\alpha}$ $\in R^{3}$ being regarded as parameters. Take $\nu<3 / 2$ and $s>3 / 2$ arbitrarily but close enough to $3 / 2$. Then the operator in question is of Hilbert-Schmidt class on $L^{2}\left(R^{3} ; d y_{\alpha}\right)$ and its norm is bounded uniformly in $x_{\alpha}$ and $E$. Hence it is also bounded uniformly in $E$ as an operator on $L^{2}\left(R^{6}\right)$. Thus, by interpolation, the lemma follows at once.

Lemma 5.2. Let $0 \leq \sigma<3 / 2$. Then

$$
X_{-s}^{\alpha}\left(H_{0}+E\right)^{-\sigma / 2} \in \mathrm{Op}\left(E^{0}\right) \text { for any } s>\sigma .
$$

Proof. Recall that the coordinates dual to $\left(x_{\alpha}, y_{\alpha}\right)$ are denoted as $\left(p_{\alpha}, q_{\alpha}\right) \in$ $R^{3 \times 2}$. If we use the Fourier transformation in $y_{\alpha}$, then the operator in the lemma is represented as the direct integral

$$
\int \oplus X_{-s}^{\alpha}\left(T^{\alpha}+\left|q_{\alpha}\right|^{2} / 2 n_{\alpha}+E\right)^{-\sigma / 2} d q_{\alpha}
$$

with $T^{\alpha}=-\Delta / 2 m_{\alpha}$. We can prove in the same way as in the proof of Lemma 5.1 that the operator in the integrand is bounded uniformly in $q_{\alpha}$ and $E$ as an operator on $L^{2}\left(R^{3} ; d x_{\alpha}\right)$. This completes the proof.

Lemma 5.3 Let $K=H_{0}+U$ be as in section 3 . Then

$$
X_{-s}^{\gamma}(K+E)^{-1} X_{-s^{\prime}}^{\delta} \in \operatorname{Op}\left(E^{0}\right)
$$

for any $s, s^{\prime}>1 / 2$ with $s+s^{\prime}>2$. The operator $K_{\alpha}$ defined by (2.4) also has the same property as above. 
Proof. Let $0 \leq \sigma<3 / 2$. We shall show that

$$
X_{-s}^{\alpha}(K+E)^{-\sigma / 2} \in \mathrm{Op}\left(E^{0}\right)
$$

for any $s>\sigma$, from which the lemma follows at once. Since $U$ is non-negative, the integral kernel of $(K+E)^{-\sigma / 2}$ is non-negative and does not exceed that of $\left(H_{0}+\right.$ $E)^{-\sigma / 2}$. Hence Lemma 5.2 implies (5.1) and the proof is complete.

Lemma 5.4. Assume that $\alpha \neq \beta$. Let $\sigma, \nu$ and $\nu^{\prime}$ be non-negative numbers with $\sigma+\nu+\nu^{\prime}<3$. Suppose that $\sigma$ has a decomposition $\sigma=\mu+\mu^{\prime}, \mu, \mu^{\prime} \geq 0$, such that $\nu+\mu<3 / 2$ and $\nu^{\prime}+\mu^{\prime}<3 / 2$. Then

$$
X_{-s}^{\alpha} \omega_{\alpha}(E)^{-\nu}\left(H_{0}+E\right)^{-\sigma / 2} \omega_{\beta}(E)^{-\nu^{\prime}} X_{-s^{\prime}}^{\beta} \in(H S)_{\varepsilon}
$$

for $s, s^{\prime}$ such that $s>\nu^{\prime}+\mu^{\prime}$ and $s^{\prime}>\nu+\mu$.

Proof. Denote by $A(E)$ the operator in the lemma. We first show that

$$
A(E) \in \mathrm{Op}\left(E^{0}\right) .
$$

To see this, we write $A(E)$ as

$$
A(E)=X_{-s}^{\alpha} \omega_{\beta}(E)^{-\left(\nu^{\prime}+\mu^{\prime}\right)}\left\{\omega_{\beta}(E)^{\mu^{\prime}}\left(H_{0}+E\right)^{-\sigma / 2} \omega_{\alpha}(E)^{\mu}\right\} \omega_{\alpha}(E)^{-(\nu+\mu)} X_{-s^{\prime}}^{\beta}
$$

according to the decomposition for $\sigma$. The operator in the brackets is obviously of class $\mathrm{Op}\left(E^{0}\right)$ and hence (5.2) follows from Lemma 5.1.

Let $\theta$ again denote the characteristic function of the interval $[0,1]$. Let $f \in$ $C_{0}\left(R^{3}\right)$ be of compact support. Denote by $f^{\alpha}$ and $f^{\beta}$ the multiplication operators by $f\left(x_{\alpha}\right)$ and $f\left(x_{\beta}\right)$, respectively. To complete the proof, it suffices by (5.2) to show that

$$
B(E)=f^{\alpha} \omega_{\alpha}(E)^{-\nu} \theta\left(H_{0}\right)\left(H_{0}+E\right)^{-\sigma / 2} \omega_{\beta}(E)^{-\nu^{\prime}} f^{\beta}
$$

is bounded uniformly in $E$ as an operator of Hilbert-Schmidt class on $L^{2}\left(R^{6}\right)$. To prove this, we consider the operator

$$
\Lambda(E)=\Psi_{\alpha} B(E) \Psi_{\beta}^{*}: L^{2}\left(R^{6} ; d x_{\beta} d q_{\beta}\right) \rightarrow L^{2}\left(R^{6} ; d x_{\alpha} d q_{\alpha}\right) .
$$

As is easily seen, $\Lambda(E)$ has an integral kernel of the form

$$
(2 \pi)^{-3} f\left(x_{\alpha}\right) \exp \left(i p_{\alpha} \cdot x_{\alpha}\right) \hat{\theta}\left(q_{\alpha}, q_{\beta}\right) G\left(q_{\alpha}, q_{\beta} ; E\right) \exp \left(-i p_{\beta} \cdot x_{\beta}\right) f\left(x_{\beta}\right)
$$

with $\hat{\theta}=\theta\left(H_{0}\left(q_{\alpha}, q_{\beta}\right)\right)$, where $p_{\alpha}$ and $p_{\beta}$ are represented as in (2.2) in terms of $\left(q_{\alpha}, q_{\beta}\right)$ and $G\left(q_{\alpha}, q_{\beta} ; E\right)$ is defined as 


$$
\left.\omega\left(\left|q_{\alpha}\right|^{2} / 2 n_{a}+E\right)^{1 / 2}\right)^{-\nu}\left(H_{0}\left(q_{\alpha}, q_{\beta}\right)+E\right)^{-\sigma / 2} \omega\left(\left(\left|q_{\beta}\right|^{2} / 2 n_{\beta}+E\right)^{1 / 2}\right)^{-\nu^{\prime}} .
$$

By assumption, it follows that the Hilbert-Schmidt norm of $\Lambda(E)$ is bounded uniformly in $E$ and hence the proof is complete.

Lemma 5.5. Assume that $\alpha \neq \beta$. Then:

(1) $X_{-\rho / 2}^{\alpha}\left(K_{\alpha}+E\right)^{-1} X_{-\rho / 2}^{\beta} \in(H S)_{\varepsilon}$.

(2) $X_{-\rho / 2}^{\alpha} \omega_{\alpha}(E)^{-1 / 2}\left(K_{\alpha}+E\right)^{-1} X_{-\rho / 2}^{\beta} \in(H S)_{\varepsilon}, \quad s>3 / 2$.

Proof. We prove only (2) for $s, 3 / 2<s<\rho-1 / 2$. A similar argument applies to (1). We denote by $B(E)$ the operator under consideration. By use of the resolvent equation, $\left(K_{\alpha}+E\right)^{-1}$ is represented as

$$
\left(K_{\alpha}+E\right)^{-1}=\left(H_{0}+E\right)^{-1}-\left(K_{\alpha}+E\right)^{-1} U_{\alpha}\left(H_{0}+E\right)^{-1} .
$$

Write $U_{\alpha}$ as $U_{\alpha}=X_{-(\rho-s)}^{\alpha} r_{0}(E) X_{-s}^{\alpha}$. Since $\omega_{\alpha}(E)$ and $K_{\alpha}$ are commutative with each other, we see from Lemma 5.3 that $B(E)$ takes the form

$$
B(E)=r_{0}(E) X_{-s}^{\alpha} \omega_{\alpha}(E)^{-1 / 2}\left(H_{0}+E\right)^{-1} X_{-\rho / 2}^{\beta} .
$$

This, together with Lemma 5.4, proves (2).

5.2. We now prove Lemma 3.2.

Proof of Lemma 3.2. (1) By the resolvent equation, we have

$$
(K+E)^{-1}=\left(K_{\alpha}+E\right)^{-1}-\sum_{\gamma \neq \alpha}\left(K_{\alpha}+E\right)^{-1} U_{r}(K+E)^{-1},
$$

where $\sum_{\gamma \neq \alpha}$ denotes the summation over two pairs except for $\alpha$. Hence, by Lemma 5.3 , the operator under consideration takes the form

$$
\sum_{\gamma \neq \alpha} r_{0}(E) X_{-\rho / 2}^{\alpha}\left(K_{\alpha}+E\right)^{-1} X_{-\rho / 2}^{\gamma} r_{0}(E)
$$

This, together with Lemma 5.5, proves (1).

(2) (2) is proved in almost the same way as in the proof of (1). We first note that $P_{1}^{\alpha} W_{\alpha}^{1 / 2} X_{s}^{\alpha}$ is bounded for any $s, 3 / 2<s<\rho-1 / 2$, by Lemma 1.2. By use of (5.4) again, we can represent the operator under consideration in the form

$$
\sum_{\gamma \neq \alpha} r_{0}(E) X_{-s}^{\alpha} \omega_{\alpha}(E)^{-1 / 2}\left(K_{\alpha}+E\right)^{-1} X_{-\rho / 2}^{\gamma} r_{0}(E)
$$

for $s$ as above. Hence (2) again follows from Lemma 5.5. 
(3) We can write $(K+E)^{-1}$ as

$$
\begin{aligned}
(K+E)^{-1}= & \left(K_{\alpha}+E\right)^{-1}-\sum_{\gamma \neq \alpha}\left(K_{\alpha}+E\right)^{-1} U_{\gamma}\left(K_{\alpha}+E\right)^{-1}, \\
& +\sum_{\gamma \neq \alpha} \sum_{\delta \neq \alpha}\left(K_{\alpha}+E\right)^{-1} U_{\gamma}(K+E)^{-1} U_{\delta}\left(K_{\alpha}+E\right)^{-1}
\end{aligned}
$$

by repeated use of (5.4). Hence (3) follows immediately from Lemmas 5.3 and 5.5.

5.3. We here prove Lemma 3.3. The proof requires the two auxiliary lemmas below.

Lemma 5.6. Let $\Gamma_{\alpha \beta}(E), \alpha \neq \beta$, be as in Lemma 3.3. Define $D_{\alpha}(E): L^{2}\left(R^{6}\right) \rightarrow$ $L^{2}\left(R^{6}\right)$ by

$$
D_{\alpha}(E)=\mathrm{Id}-\left(K_{\alpha}+E\right)^{-1} U_{\alpha}
$$

Then one has

$$
Q_{\alpha \beta}^{f}(E) \sim P_{1}^{\alpha} W_{\alpha}^{1 / 2} D_{\alpha}(E) f_{\alpha}(E)^{-1 / 2} \Gamma_{\alpha \beta}(E) f_{\beta}(E)^{-1 / 2} D_{\beta}(E)^{*} W_{\beta}^{1 / 2} P_{1}^{\beta} .
$$

Proof. We write $(K+E)^{-1}$ as in (5.5);

$$
(K+E)^{-1}=B_{0}(E)+B_{1}(E)+B_{2}(E),
$$

where

$$
\begin{aligned}
& B_{0}(E)=\left(K_{\alpha}+E\right)^{-1}\left(\mathrm{Id}-U_{\beta}\left(K_{\beta}+E\right)^{-1}\right), \\
& B_{1}(E)=-\left(K_{\alpha}+E\right)^{-1} U_{\gamma}\left(K_{\beta}+E\right)^{-1}, \quad \gamma \neq \alpha, \beta, \\
& B_{2}(E)=\sum_{\gamma \neq \alpha} \sum_{\delta \neq \beta}\left(K_{\alpha}+E\right)^{-1} U_{\gamma}(K+E)^{-1} U_{\delta}\left(K_{\beta}+E\right)^{-1} .
\end{aligned}
$$

We can further rewrite $B_{0}(E)$ as

$$
B_{0}(E)=D_{\alpha}(E)\left(H_{0}+E\right)^{-1} D_{\beta}(E)^{*}
$$

by making use of the relation $\left(K_{\alpha}+E\right)^{-1}=D_{\alpha}(E)\left(H_{0}+E\right)^{-1}$, which follows from the resolvent equation at once. Hence the lemma is obtained as an immediate consequence of Lemmas 5.3 and 5.5.

Lemma 5.7. Let $s$ be such that $3 / 2<s<\rho-1 / 2$ and let $D_{0 \alpha}$ and $D_{\alpha}(E)$ be as in Lemmas 3.3 and 5.6, respectively. Then there exists $\nu>0$ such that 


$$
X_{-s}^{\alpha}\left(D_{\alpha}(E)-D_{0 \alpha}\right) X_{s}^{\alpha} \omega_{\alpha}(E)^{-\nu} \in \mathrm{Op}\left(E^{0}\right)
$$

Proof. Let $s$ be as above. Then we can prove in the same way as in the proof of Lemma 4.2 that

$$
\left\|X_{-s}^{\alpha}\left(R\left(d^{2} ; K^{\alpha}\right)-R\left(0 ; K^{\alpha}\right)\right) X_{-(\rho-s)}^{\alpha}\right\|=O\left(d^{\nu}\right), \quad d \rightarrow 0,
$$

for some $\nu>0$ as an operator acting on $L^{2}\left(R^{3} ; d x_{\alpha}\right)$. This proves the lemma.

Proof of Lemma 3.3. Recall the definition (3.8) of $f_{\alpha}(E)$. Since $f(s)$ behaves as in (3.5), the lemma is obtained by combining Lemmas $1.2,5.4,5.6$ and 5.7.

\section{\$6. Proof of Theorem 2}

Theorem 2 can be verified in almost the same way as in the proof of Theorem 1 , so we give only a sketch for the proof.

(1) We follow the same notations used in the proof of Theorem 1. Let $H(\lambda)$, $0<\lambda \ll 1$, be defined by $(0.2)$. Recall that $N_{0}(\lambda)$ denotes the number of negative eigenvalues of $H(\lambda)$. It is convenient to rewrite $H(\lambda)$ as

$$
H(\lambda)=H-\lambda V=(1-\lambda)\left(H+\kappa H_{0}\right)
$$

with $\kappa=\lambda /(1-\lambda)$. We denote by $N_{E}(\kappa), 0<E \ll 1$, the number of negative eigenvalues less than $-E$ of $H+\kappa H_{0}$ and write, in particular, $N_{0}(\kappa)$ for $N_{0}(\lambda)$. We apply the Birman-Schwinger principle to $H+\kappa H_{0}=K+\kappa H_{0}-W$. Then we obtain $N_{E}(\kappa)=n(1 ; M(\kappa, E))$, where $\left.M(\kappa, E)\right): L^{2}\left(R^{6}\right) \rightarrow L^{2}\left(R^{6}\right)$ is defined by $M(\kappa, E)=\sum_{\alpha} M_{\alpha}(\kappa, E){ }^{*} M_{\alpha}(\kappa, E)$ with

$$
M_{\alpha}(E, \kappa)=W_{\alpha}^{1 / 2}\left(K+\kappa H_{0}+E\right)^{-1 / 2} .
$$

(2) We again write $T^{\alpha}$ for $-\Delta / 2 m_{\alpha}$, so that $H_{0}=T^{\alpha}+T_{\alpha}$. The second step is to study the behavior as $d \rightarrow 0$ of the operator

$$
A\left(d ; K^{\alpha}\right)=\mathrm{Id}-W_{\alpha}^{1 / 2}\left(K^{\alpha}+\kappa T^{\alpha}+d^{2}\right)^{-1} W_{\alpha}^{1 / 2}, \quad d \geq 0 .
$$

To do this, we write the inverse $\left(K^{\alpha}+\kappa T^{\alpha}+d^{2}\right)^{-1}$ as

$$
R\left(d^{2} ; K^{\alpha}\right)^{1 / 2}\left(\mathrm{Id}+\kappa R\left(d^{2} ; K^{\alpha}\right)^{1 / 2} T^{\alpha} R\left(d^{2} ; K^{\alpha}\right)^{1 / 2}\right)^{-1} R\left(d^{2} ; K^{\alpha}\right)^{1 / 2}
$$

with $R\left(d^{2} ; K^{\alpha}\right)=\left(K^{\alpha}+d^{2}\right)^{-1}$. Since both the operators $R\left(d^{2} ; K^{\alpha}\right)^{1 / 2}\left(T^{\alpha}\right)^{1 / 2}$ and $R\left(d^{2} ; K^{\alpha}\right)^{1 / 2} W_{\alpha}^{1 / 2}$ are bounded uniformly in $d$ as an operator on $L^{2}\left(R^{3} ; d x_{\alpha}\right)$, the Neumann series expansion yields 


$$
A_{\kappa}\left(d ; K^{\alpha}\right)=A\left(d ; K^{\alpha}\right)+\kappa W_{\alpha}^{1 / 2} R\left(d^{2} ; K^{\alpha}\right) T^{\alpha} R\left(d^{2} ; K^{\alpha}\right) W_{\alpha}^{1 / 2}+\mathrm{Op}\left(\kappa^{2}\right),
$$

where $A\left(d ; K^{\alpha}\right)$ is defined by $(2.5)$ and $O p\left(\kappa^{2}\right)$ denotes a bounded operator with its operator norm of order $O\left(\kappa^{2}\right)$ as $\kappa \rightarrow 0$ uniformly in $d$. Note that

$$
T^{\alpha} \geq\left|x_{\alpha}\right|^{-2} / 4 \geq c W_{\alpha}
$$

for some $c>0$ in the form sense. Hence we obtain from Lemma 1.3 that for $d>0$ small enough,

$$
\begin{aligned}
& A_{\kappa}\left(d ; K^{\alpha}\right) \geq \sigma_{\alpha}\left(d f(d)+c_{+} \kappa\right) P_{1}^{\alpha}+c_{2} P_{2}^{\alpha}, \\
& A_{\kappa}\left(d ; K^{\alpha}\right) \leq \sigma_{\alpha}\left(d g(d)+c_{-} \kappa\right) P_{1}^{\alpha}+c_{2}^{\prime} P_{2}^{\alpha}
\end{aligned}
$$

with positive constants $c_{ \pm}, c_{2}$ and $c_{2}^{\prime}$, where $f(d)$ and $g(d)$ are strictly positive functions having the same property as in (3.5). We here note that the argument below does not require the explicit values of constants $c_{ \pm}$.

(3) Relation (6.1) enables us to repeat the same argument as in the proof of Theorem 1 and we have

$$
n\left((1+\varepsilon) ; \mathscr{g}_{-}(\kappa, E)\right)-C_{\varepsilon} \leq N_{E}(\kappa) \leq n\left((1-\varepsilon) ; \mathscr{g}_{+}(\kappa, E)\right)+C_{\varepsilon}
$$

for any $\varepsilon>0$ small enough, where $\mathscr{g}_{ \pm}(\kappa, E): \mathscr{L}^{2}\left(B_{1}\right) \rightarrow \mathscr{L}^{2}\left(B_{1}\right)$ is the $3 \times 3$ matrix with $J_{\alpha \beta}^{ \pm}(\kappa, E), \alpha \neq \beta$, as the off-diagonal entries and $J_{\alpha \alpha}^{ \pm}(E)=0$ as the diagonal ones. The integral kernel $J_{\alpha \beta}^{ \pm}\left(q_{\alpha}, q_{\beta} ; \kappa, E\right)$ of the operator $J_{\alpha \beta}^{ \pm}(\kappa, E)$ : $L^{2}\left(B_{1} ; d q_{\beta}\right) \rightarrow L^{2}\left(B_{1} ; D q_{\alpha}\right)$ takes the form

$$
\tau_{\alpha \beta} \zeta_{\alpha}^{ \pm}\left(q_{\alpha} ; \kappa, E\right)\left((1+\kappa) H_{0}\left(q_{\alpha}, q_{\beta}\right)+E\right)^{-1} \zeta_{\beta}^{ \pm}\left(q_{\beta} ; \kappa, E\right),
$$

where $\tau_{\alpha \beta}$ is as in (3.12) and $\zeta_{\alpha}^{ \pm}\left(q_{\alpha} ; \kappa, E\right)$ is defined by

$$
\zeta_{\alpha}^{ \pm}\left(q_{\alpha} ; \kappa, E\right)=\chi\left(q_{\alpha}\right)\left(\left|q_{\alpha}\right|^{2} / 2 n_{\alpha}+E+c_{ \pm}^{2} \kappa^{2}\right)^{-1 / 4} .
$$

Let $J_{\alpha \beta}(E): L^{2}\left(B_{1} ; d q_{\beta}\right) \rightarrow L^{2}\left(B_{1} ; d q_{\alpha}\right)$ be as in the proof of Theorem 1. Recall the representation (3.12) for its integral kernel. As is easily seen, the Hilbert-Schmidt norm of the difference between $J_{\alpha \beta}^{ \pm}(\kappa, 0)$ and $J_{\alpha \beta}\left(c_{ \pm}^{2} \kappa^{2}\right)$ is bounded uniformly in $\kappa$. Hence we let $E \rightarrow 0$ in (6.2) to obtain that

$$
n\left((1+\varepsilon) ; \mathscr{g}_{0}\left(c_{-}^{2} \kappa^{2}\right)\right)-C_{\varepsilon} \leq N_{0}(\kappa) \leq n\left((1-\varepsilon) ; \mathscr{g}_{0}\left(c_{+}^{2} \kappa^{2}\right)\right)+C_{\varepsilon}
$$

with another $C_{\varepsilon}$ independent of $\kappa$. This, together with Lemma 3.4, proves the theorem. 


\section{\$7. Concluding remark}

As previously stated, the three-body Hamiltonian $H$ has in general an infinite number of negative eigenvalues accumulating at zero except for a certain special case, if only two subsystem Hamiltonians have a resonance state at zero energy. In such a case also, we can derive the asymptotic formula for $N(E)$ or $N_{0}(\lambda)$ with another leading coefficient $C_{0}$. In the present section, we shall discuss this problem briefly.

For notational brevity, we fix the three pairs $\alpha, \beta$ and $\gamma$ as $\alpha=(1,2)$, $\beta=(1,3)$ and $\gamma=(2,3)$, respectively, and assume that the two-body subsystem Hamiltonians $H^{\alpha}$ and $H^{\beta}$ have a resonance state at zero energy. We further assume that the mass $m_{1}$ is finite; $m_{1}<\infty$. Under these assumptions, we can show that $H$ has an infinite number of negative eigenvalues. However, if $m_{1}$ is infinite, then the Efimov effect is not in general expected to occur. In fact, if the pair potential $V_{r}=V_{23}$ is non-negative, then it follows from assumption (H.1) that $H \geq 0$ and hence $H$ cannot have any negative eigenvalue.

We consider only the problem on the asymptotics for $N(E)$. A similar argument applies to $N_{0}(\lambda)$. By repeating the same argument as in the proof of Theorem 1 , we can reduce the problem under consideration to study on the eigenvalue asymptotics for the integral operator

$$
g_{1}(E)=\left(\begin{array}{ccc}
0 & J_{\alpha \beta}(E) & 0 \\
J_{\beta \alpha}(E) & 0 & 0 \\
0 & 0 & 0
\end{array}\right): L^{2}\left(B_{1}\right) \rightarrow L^{2}\left(B_{1}\right)
$$

with entry $J_{\alpha \beta}(E): L^{2}\left(B_{1} ; d q_{\beta}\right) \rightarrow L^{2}\left(B_{1} ; d q_{\alpha}\right)$ whose integral kernel is given by (3.12). As a result, we obtain

$$
n\left((1+\varepsilon) ; \mathscr{g}_{1}(E)\right)-C_{\varepsilon} \leq N(E) \leq n\left((1-\varepsilon) ; \mathscr{g}_{1}(E)\right)+C_{\varepsilon} .
$$

According to the results in [7] again, we know that the limit

$$
\Theta_{1}(\mu)=\lim _{E \rightarrow 0} n\left(\mu: \mathscr{J}_{1}(E)\right) /|\log E|
$$

exists as a continuous function of $\mu>0$ and that the constant $C_{1}=\Theta_{1}(1)$ is evaluated from below as

$$
C_{1} \geq 8^{-1} \pi^{-1} \log \mu_{\alpha \beta}, \quad \mu_{\alpha \beta}=\left(n_{\alpha} / m_{a}\right)\left(n_{\beta} / m_{\beta}\right) .
$$

It should be noted that $\mu_{\alpha \beta}>1$ under the finiteness assumption of mass $m_{1}$ and hence $C_{1}>0$. Thus we can derive the asymptotic formula 


$$
N(E)=C_{1}|\log E|(1+o(1)), \quad E \rightarrow 0 .
$$

In the special case with identical masses, the constant $C_{1}$ is explicitly determined as $C_{1}=\lambda / 2 \pi$ with the unique positive $\operatorname{root} \lambda>0$ of the equation

$$
\lambda=2^{2} \cdot 3^{-1 / 2}(\sinh \pi \lambda / 6) /(\cosh \pi \lambda / 2) .
$$

\section{REFERENCES}

[1] V. Efimov, Energy levels arising from resonant two-body forces in a three-body systems, Phys. Lett., B 33 (1970), 563-564.

[2] A. Jensen and T. Kato, Spectral properties of Schrödinger operators and time-decay of the wave functions, Duke Math. J., 46 (1979), 583-611.

[ 3 ] R. G. Newton, Scattering Theory of Waves and Particles, 2nd ed., Springer Verlag, 1982.

[ 4 ] Y. N. Ovchinnikov and I. M. Sigal, Number of bound states of three-body systems and Efimov's effect, Ann. Phys., 123 (1979), 274-295.

[5] M. Reed and B. Simon, Methods of Modern Mathematical Physics IV, Analysis of Operators, Academic Press, 1982.

[6] B. Simon, Large time behavior of the $L^{p}$ norm of Schrödinger semigroups, J. Funct. Anal., 40 (1981), 66-83.

[7] A. V. Sobolev, The Efimov effect: Discrete spectrum asymptotics, preprint, University of Toronto (1992).

[8] H. Tamura, The Efimov effect of three-body Schrödinger operators, J. Funct. Anal., 95 (1991), 433-459.

[9] D. R. Yafaev, On the theory of the discrete spectrum of the three-particle Schrödinger operator, Math. USSR-Sb, 23 (1974), 535-559.

Department of Mathematics

Ibaraki University

Mito, Ibaraki, 310 Japan 